\title{
A NOTE ON ANGULAR REPRESENTATIONS FOR SCATTERED WAVES*
}

\author{
$\mathrm{Br}$ \\ DAN CENSOR \\ Tel-Aviv University
}

\begin{abstract}
Differential-operator representations for the scattered field were given by Twersky [1], [3], [4], and Burke, Censor and Twersky [2]. Essentially, from the data provided on a circle (in two dimensions) or a sphere (in three dimensions), the field may be reconstructed for arbitrary distances.

Presently reconstruction of the field is considered from data provided in other regions of space, e.g., on a ray for the two-dimensional case. These representations facilitate the investigation of velocity-dependent scattering by collecting the data near the path of the moving object.
\end{abstract}

Introduction. The time-harmonic two-dimensional scalar scattered field is given, e.g., in terms of the special functions representation,

$$
u(\mathbf{r}, t)=\sum_{m=-\infty}^{\infty} i^{m} A_{m} H_{m}(k r) e^{i m \phi-i \omega t}, \quad r>\rho .
$$

where $A_{m}$ are coefficients, $H_{m}(k r)=H_{m}^{(1)}(k r)$ is the Hankel function of the first kind of order $m$, and $\rho$ is the radius of the circle circumscribing the scatterer. Twersky [1], and Burke, Censor and Twersky [2] (who give a comprehensive review of earlier relevant results by Sommerfeld, Wilcox and Karp), recast $H_{m}$ as an asymptotic [1] or an exact [2] series in powers of $\mathrm{m}^{2}$, and replace it by a differential operator acting on $g(\phi)$, the scattering amplitude

$$
g(\phi)=\sum_{m=-\infty}^{\infty} A_{m} e^{i m \phi} .
$$

Thus to obtain the asymptotic representation $H_{m}(k r)$ is replaced in (1) by the series

$$
\begin{gathered}
H(k r) \sum_{\nu=0} \frac{\left(1-4 m^{2}\right)\left(9-4 m^{2}\right) \cdots\left([2 \nu-1]^{2}-4 m^{2}\right)}{(i 8 k r)^{\nu} \nu !} \equiv D\left(k r ;-m^{2}\right), \\
H(k r)=(2 / i \pi k r)^{1 / 2} e^{i k r},
\end{gathered}
$$

and it is noted that

$$
\left(m^{2}-\partial_{\phi}^{2}\right) e^{i m} \equiv 0 .
$$

Consequently (1) can be represented by

$$
u \sim e^{-i \omega t} D\left(k r ; \partial_{\phi}^{2}\right) g(\phi) .
$$

* Received December 23, 1969; revised version received May 30, 1970. 
If $H(k r) g(\phi)$ is known, i.e. if the far-zone field is specified, then by means of (5) higher powers of $1 / k r$ might be obtained for nearer regions.

The exact representation [2] is given by

$$
\begin{aligned}
u & =\left[H_{0}(k r)\left(R_{e} g_{e}+R_{0}^{\prime} g_{0}\right)+H_{1}(k r)\left(R_{0} g_{0}+R_{e}^{\prime} g_{e}\right)\right] e^{-i \omega t}, \\
g_{e o} & =\frac{1}{2}[g(\phi) \pm g(\pi+\phi)] \\
R_{e} & =1-\frac{\partial^{2}\left(\partial^{2}+4\right)}{2(2 p)^{2}}+\frac{\partial^{2}\left(\partial^{2}+4\right)^{2}\left(\partial^{2}+16\right)}{4 !(2 p)^{4}}+\cdots, \\
R_{e}^{\prime} & =\frac{\partial^{2}}{2 p}-\frac{\partial^{2}\left(\partial^{2}+4\right)^{2}}{3 !(2 p)^{3}}+\frac{\partial^{2}\left(\partial^{2}+4\right)^{2}\left(\partial^{2}+16\right)^{2}}{5 !(2 p)^{5}}+\cdots, \\
R_{0}^{\prime} / i & =-\frac{\partial^{2}+1}{2 p}+\frac{\left(\partial^{2}+9\right)\left(\partial^{2}+1\right)^{2}}{3 !(2 p)^{3}}-\frac{\left(\partial^{2}+25\right)\left(\partial^{2}+9\right)^{2}\left(\partial^{2}+1\right)^{2}}{5 !(2 p)^{5}}+\cdots, \\
R_{0} / i & =1-\frac{\left(\partial^{2}+1\right)^{2}}{2(2 p)^{2}}+\frac{\left(\partial^{2}+1\right)\left(\partial^{2}+9\right)^{2}}{4 !(2 p)^{4}}+\cdots, \\
\partial & \equiv \partial / \partial \phi, \quad p=k r .
\end{aligned}
$$

For three-dimensional vector waves Twersky [3] obtains a dyadic differential operator,

$$
\begin{aligned}
& \mathbf{u}= e^{-i \omega t} h(k r) \tilde{D}(k r ; \tilde{D}) \cdot \mathbf{g}(\hat{r}), \\
& \widetilde{D}(k r ; \widetilde{D})=\sum_{\nu=0}^{\infty}\left(\frac{i}{2 k r}\right)^{\nu} \frac{1}{\nu !} \widetilde{D} \cdot(\tilde{D}-1 \cdot 2 \tilde{I}) \cdot(\widetilde{D}-2 \cdot 3 \tilde{I}) \cdots(\widetilde{D}-[\nu-1] \nu \tilde{I}), \\
& h(k r)=e^{i k r} / i k r, \\
& \widetilde{D} \cdot \mathbf{g}= \hat{\mathbf{r}}\left\{D g_{r}+2 g_{r}+\frac{2}{\sin \theta}\left[\partial_{\theta}\left(\sin \theta g_{\theta}\right)+\partial_{\phi} g_{\phi}\right]\right\} \\
&+\hat{\boldsymbol{\theta}}\left\{D g_{\theta}+\frac{1}{\sin ^{2} \theta}\left[g_{\theta}+2 \cos \theta \partial_{\phi} g_{\phi}\right]-2 \partial_{\theta} g_{r}\right\} \\
&+\hat{\boldsymbol{\phi}}\left\{D g_{\phi}-\frac{1}{\sin ^{2} \theta}\left[-g_{\phi}+2 \cos \theta \partial_{\phi} g_{\theta}\right]-\frac{2}{\sin \theta} \partial_{\phi} g_{r}\right\}, \\
& D=-\frac{1}{\sin ^{2} \theta}\left[\partial_{\phi}^{2}+\sin \theta \partial_{\theta}\left(\sin \theta \partial_{\theta}\right)\right],
\end{aligned}
$$

where $\tilde{I}$ is the idemfactor dyadic. For the Cartesian components of $\mathrm{g}, \tilde{D}$ reduces to $D \tilde{I}$, where $D$ is the Beltrami operator; hence (7) contains the three-dimensional scalar case given before [4].

Two dimensions. Using the same approach, consider the two-dimensional problem of reconstructing the scattered field from the data given on a ray $\phi=0$. For the even part of the field we write

$$
u_{e}=[u(\phi)+u(-\phi)] / 2=e^{-i \omega t} \sum_{n} i^{m} A_{m} H_{m}(k r) \cos m \phi .
$$

By means of Bessel's differential equation, which is satisfied by $H_{m}(k r)$, a differential operator is defined,

$$
B_{r} H_{m} \equiv\left[k r \partial_{k r}\left(k r \partial_{k r}\right)+k^{2} r^{2}\right] H_{m}(k r)=m^{2} H_{m} .
$$


This operator $B_{r}$ may replace $m^{2}$, and since it is independent of $m$, it can be taken outside the summation symbol. Now recast $\cos m \phi$ in powers of $m^{2}$; this yields

$$
\begin{aligned}
u_{e} & =e^{-i \omega t} \sum_{m}\left[1-\frac{m^{2} \phi^{2}}{2 !}+\frac{m^{4} \phi^{4}}{4 !}+\cdots\right] i^{m} A_{m} H_{m} \\
& =e^{-i \omega t}\left[1-\frac{\phi^{2}}{2 !} B_{r}+\frac{\phi^{4}}{4 !} B_{r}^{2}+\cdots\right] \sum_{m} i^{m} A_{m} H_{m} \\
& \left.\equiv e^{-i \omega t} \cdot D_{e}\left(B_{r} ; \phi\right) u\right|_{\phi=0},
\end{aligned}
$$

where $B_{r}^{2}=B_{r} B_{r}$ etc., $\left.u\right|_{\phi=0}$ is the field measured on $\phi=0$.

The derivative with respect to $\phi$ of the odd part of the field again involves $\cos m \phi$

$$
\partial_{\phi} u_{0} \equiv \partial_{\phi}[u(\phi)-u(-\phi)] / 2=e^{-i \omega t} \sum_{m} i^{m} H_{m} A_{m} i m \cos m \phi .
$$

Therefore, similarly to (10),

$$
\partial_{\phi} u_{0}=e^{-i \omega t} D_{e}\left(B_{r} ; \phi\right) \sum_{m} i^{m} H_{m} i m A_{m}=\left.e^{-i \omega t} D_{e}\left(\partial_{\phi} u\right)\right|_{\phi-0} .
$$

Writing

$$
D_{e}=\partial_{\phi}\left(\phi-\frac{B_{r} \phi^{3}}{3 !}+\frac{B_{r}^{2} \phi^{5}}{5 !}+\cdots\right) \equiv \partial_{\phi} D_{0}\left(B_{\tau} ; \phi\right),
$$

and integrating both sides of (12) with respect to $\phi$ between the same limits yields

$$
u_{0}=\left.e^{-i \omega t} D_{0}\left(B_{r} ; \phi\right)\left(\partial_{\phi} u\right)\right|_{\phi-0} \text {. }
$$

Consequently, in order to find $u_{0}$ it is necessary to measure on $\phi=0 \partial_{\phi} u$; for $u_{e}$ the field itself suffices. Summing up, we have,

$$
u=u_{e}+u_{0}=e^{-i \omega t}\left[\left.D_{e}\left(B_{r} ; \phi\right) u\right|_{\phi=0}+\left.D_{0}\left(B_{r} ; \phi\right)\left(\partial_{\phi} u\right)\right|_{\phi-0}\right] .
$$

Three dimensions. The solution of the scalar scattering problem in three dimensions is

$$
u=\sum_{n=0}^{\infty} \sum_{m=-n}^{n} i^{n} a_{n m} h_{n}(k r) P_{n}^{m}(\cos \theta) e^{i m \phi-i \omega t},
$$

where $h_{n}, P_{n}^{m}$ denote spherical Hankel functions and associated Legendre polynomials, respectively. The analogue of (9) is obtained by defining an operator $B_{r, \theta}$ acting on $h_{n}(k r) P_{n}^{m}(\cos \theta)$,

$$
B_{r, \theta} \equiv \sin ^{2} \theta \partial_{\tau}\left(r^{2} \partial_{r}\right)+\sin \theta \partial_{\theta}\left(\sin \theta \partial_{\theta}\right)+k^{2} r^{2} \sin ^{2} \theta=m^{2},
$$

implied by separation of the scalar wave equation in spherical coordinates. Similarly to $(15)$ we get

$$
u=u_{e}+u_{0}=e^{-i \omega t}\left[\left.D_{e}\left(B_{r, \theta} ; \phi\right) u\right|_{\phi=0}+\left.D_{0}\left(B_{r, \theta} ; \phi\right)\left(\partial_{\phi} u\right)\right|_{\phi=0} .\right.
$$

The Cartesian components of the electromagnetic field vectors satisfy the scalar wave equation, hence for vector waves substituting $u$ for $u$ in (18) yields the desired representation.

Consider now the case of scalar scattering by an obstacle rotationally symmetric about the direction of incidence of the exciting plane wave. For this case the scattered field is given by 


$$
u=\sum_{n=0}^{\infty} i^{n} A_{n} P_{n}(\cos \theta) h_{n}(k r) e^{-i \omega t}
$$

i.e., there is no dependence on $\phi$. We define a spherical Bessel operator analogous to (9) acting on $h_{n}(k r)$ :

$$
A_{r} \equiv k^{2} r^{2} \partial_{k r}^{2}+2 k r \partial_{k r}+k^{2} r^{2}=n(n+1) \equiv d .
$$

The Legendre polynomials $P_{n}(\cos \theta)$ may be written (e.g., see Hobson [5]) as a series in powers of $d$,

$$
\begin{aligned}
P_{n}(\cos \theta) & =1-\frac{d}{(1 !)^{2}} \sin ^{2} \frac{\theta}{2}+\frac{d(d-1 \cdot 2)}{(2 !)^{2}} \sin ^{4} \frac{\theta}{2}+\cdots \\
& =F\left(n+1,-n ; 1 ; \sin ^{2} \frac{\theta}{2}\right) \equiv E(d ; \theta) .
\end{aligned}
$$

It therefore follows that

$$
u=e^{-i \omega t} E\left(A_{r} ; \theta\right) \sum_{n=0}^{\infty} i^{n} A_{n} h_{n}(k r) .
$$

But $P_{n}(1)=1$; hence the sum in (22) is the value of the field on the ray $\theta=0$,

$$
u=\left.e^{-i \omega t} E\left(A_{r} ; \theta\right) u\right|_{\theta=0} \text {. }
$$

Therefore, by means of (23) the field at an arbitrary point may be reconstructed from data provided on the line $\theta=0$, i.e. the direction of incidence. Obviously the method fails for $P_{n}^{m}, m \neq 0$, which vanish at $\theta=0$. Indeed, we could not expect to learn about a field depending on the azimuthal direction from that on $\theta=0$, where the angle $\phi$ is not defined.

Another possible arrangement is to provide the data on the plane $\theta=\pi / 2$. For this case we exploit the representations [6]

$$
\begin{aligned}
P_{n}(\mu)= & (-1)^{n / 2} \frac{n !}{2^{n}\left(\frac{n}{2} !\right)^{2}} F\left(-\frac{n}{2}, \frac{n+1}{2} ; \frac{1}{2} ; \mu^{2}\right) \\
= & (-1)^{n / 2} \frac{1 \cdot 3 \cdot 5 \cdots(n-1)}{2 \cdot 4 \cdots n} \\
& \cdot\left[1-\frac{d}{2 !} \mu^{2}+\frac{d(d-2 \cdot 3)}{4 !} \mu^{4}-\frac{d(d-2 \cdot 3)(d-4 \cdot 5)}{6 !} \mu^{6}+\cdots\right] \\
\equiv & (-1)^{n / 2} \frac{1 \cdot 3 \cdot 5 \cdots(n-1)}{2 \cdot 4 \cdots n} G_{e}(d ; \theta),
\end{aligned}
$$

for even $n$, and

$$
\begin{aligned}
P_{n}(\mu) & =(-1)^{(n-1) / 2} \frac{n !}{2^{n-1}\left(\frac{n-1}{2} !\right)^{2}} \mu F\left(-\frac{n-1}{2}, \frac{n}{2}+1 ; \frac{3}{2} ; \mu^{2}\right) \\
& =(-1)^{(n-1) / 2} \frac{3 \cdot 5 \cdots n}{2 \cdot 4 \cdots(n-1)}\left[\mu-\frac{(d-1 \cdot 2)}{3 !} \mu^{3}+\frac{(d-1 \cdot 2)(d-3 \cdot 4)}{5 !} \mu^{5}-\cdots\right] \\
& \equiv(-1)^{(n-1) / 2} \frac{3 \cdot 5 \cdots n}{2 \cdot 4 \cdots(n-1)} G_{0}(d ; \theta)
\end{aligned}
$$


for odd $n$. From (24), (25) it is clear that $P_{n}(\mu)$ for odd $n$ vanishes at $\theta=\pi / 2$, while the even order functions exist, and vice versa for $d_{\mu} P_{n}(\mu)$. Upon writing (19) as

$$
u=u_{0}+u_{0}
$$

where $u_{e}$ contains $P_{n}$ of even order $n$, etc., we get

$$
\begin{aligned}
u_{e} & =G_{e}\left(A_{r} ; \theta\right) \sum_{n=0}^{\infty} i^{n} A_{n} h_{n}(k r)(-1)^{n / 2} \frac{1 \cdot 3 \cdot 5 \cdots(n-1)}{2 \cdot 4 \cdots n} \\
& =G_{e}\left(A_{r} ;\left.\theta U_{\bullet}\right|_{\theta-\pi / 2}\right. \\
& =\left.\left[1-\frac{A_{r}}{2 !} \cos ^{2} \theta+\frac{A_{r}\left(A_{r}-2 \cdot 3\right)}{4 !} \cos ^{4} \theta-\cdots\right] u\right|_{\theta-\pi / 2}, \quad n=0,2,4, \cdots .
\end{aligned}
$$

Here $\left.u_{\theta}\right|_{\theta-x / 2}=\left.u\right|_{\theta-\pi / 2}$ has been used. Similarly

$$
\begin{aligned}
u_{0} & =G_{0}\left(A_{r} ; \theta\right) \sum_{n=1}^{\infty} i^{n} A_{n} h_{n}(k r)(-1)^{(n-1) / 2} \frac{3 \cdot 5 \cdots n}{2 \cdot 4 \cdots(n-1)} \\
& =\left.G_{0}\left(A_{r} ; \theta\right)\left(\partial_{\mu} u_{0}\right)\right|_{\theta-\pi / 2} \\
& =\left.\left[\cos \theta-\frac{\left(A_{r}-1 \cdot 2\right)}{3 !} \cos ^{3} \theta+\frac{\left(A_{r}-1 \cdot 2\right)\left(A_{r}-3 \cdot 4\right)}{5 !} \cos ^{5} \theta-\cdots\right]\left(\partial_{\mu} u\right)\right|_{\theta-\mathbf{r} / 2} \\
& n=1,3, \cdots .
\end{aligned}
$$

Finally, using the fact that $-\partial_{\theta} / \sin \theta=\partial_{\mu}=-\partial_{\theta}$ at $\pi / 2$, we have

$$
u=\left.G_{\theta}\left(A_{r} ; \theta\right) u\right|_{\theta=\mathbf{r} / 2}-\left.G_{0}\left(A_{r} ; \theta\right)\left(\partial_{\theta} u\right)\right|_{\theta=\mathrm{r} / 2} \text {. }
$$

Hence from the knowledge of $u$ and $\partial_{\theta} u$ at $\theta=\pi / 2$ the field may be reconstructed for arbitrary points in space.

The general solution of the scalar wave equation in spherical coordinates is given by (16) where

$$
P_{n}^{m}(\mu)=\left(1-\mu^{2}\right)^{m / 2} d_{\mu}^{m} P_{n}(\mu) .
$$

The representation of $\sin ^{m} \theta$ as a series in $m$ is too complicated to be practical; therefore, instead of pulling $\sin ^{m} \theta$ out of the double summation sign (16), the data will be first Fourier-analysed with respect to $e^{i m \phi}$. Accordingly one isolates $u_{m}$ for a certain index $m$,

$$
u_{m}=\sum_{n=0}^{\infty} i^{n} A_{n m} h_{n}(k r) P_{n}^{m}(\cos \theta) e^{-i \omega t} .
$$

Now by differentiation of $G_{e}(d ; \theta), G_{0}(d ; \theta)$ with respect to $\mu$. The operator equivalent to $P_{n}^{m}$ is obtained. Thus, for example, by differentiating in (24) $G_{e}(d ; \theta) m$ times, where $m$ is even, say, we get

$$
\begin{gathered}
d_{\mu}^{m} G_{c}(d ; \theta)=(-1)^{m}\{[d-(m-1)(m-2)][d-(m-3)(m-4)] \cdots(d-2 \cdot 3) d\} \\
\cdot\left\{1-\frac{\mu^{2}}{2 !}[d-m(m+1)]+\frac{\mu^{4}}{4 !}[d-m(m+1)][d-(m+2)(m+3)]-\cdots\right\},
\end{gathered}
$$

but the operator $A_{r}$ is substituted for $d$ only in the second braces (32); the expression in the first braces is left within the sum (31). By subjecting $G_{0}(d ; \theta)$ of $(25)$ to the same process and noting that for $m=$ even the structure with respect to $\mu$ is preserved, we can write by inspection 


$$
\begin{aligned}
u_{m}= & \left.G_{e}^{(e)}\left(A_{r} ; \theta\right) u_{m}\right|_{\theta-x / 2}-\left.G_{0}^{(e)}\left(A_{r} ; \theta\right)\left(\partial_{\theta} u_{m}\right)\right|_{\theta-x / 2}, \\
G_{e}^{(e)}= & \sin ^{m} \theta\left\{1-\frac{A_{\tau}-m(m+1)}{2 !} \mu^{2}\right. \\
& \left.+\frac{\left[A_{\tau}-m(m+1)\right]\left[A_{r}-(m+2)(m+3)\right]}{4 !} \mu^{4}-\cdots\right\}, \\
G_{0}^{(e)}= & \sin ^{m} \theta\left\{\mu-\frac{A_{r}-\frac{(m+1)(m+2)}{3 !} \mu^{3}}{}\right. \\
& +\frac{\left[A_{\tau}-(m+1) \frac{(m+2)]\left[A_{\tau}-(m+3)(m+4)\right]}{5 !} \mu^{5}-\cdots\right\},}{}
\end{aligned}
$$

where the superscript (e) denotes that $m$ is even. In particular for $m=0$ we have (29) once again. For odd $m$, differentiating $G_{e}$ and $G_{0},(24)$ and (25), respectively, and disregarding operators common to all terms in a series (these are left inside the summation sign, as before), we get

$$
G_{o}^{(0)}=G_{0}^{(e)}, \quad G_{0}^{(0)}=G_{e}^{(e)},
$$

Consequently for both $m$ odd and even the first line of (33) applies. The case $m=1$ is of special interest for scattering of a plane electromagnetic wave, when $\theta=0$ is chosen as the direction of incidence, as for example in the problem of the sphere [7]. Again, the Cartesian components of the electromagnetic fields satisfy the scalar wave equation; therefore in (33) $u_{m}$ can be replaced by the vector function $u_{m}$.

Application to velocity-dependent scattering. Scattering of electromagnetic waves by arbitrary objects moving uniformly in free space has been considered by Censor [8, 9, 10]. Consider the case of a cylinder of arbitrary cross-section, at rest in frame of reference $\Gamma^{\prime}$. The incident plane electromagnetic wave is defined in the observer's proper frame $\Gamma$. Observed from $\Gamma$, frame $\Gamma^{\prime}$ is seen to move with velocity $\nabla=v \hat{\mathbf{x}}$ (i.e., in direction $\phi=0$ ). Thus in $\Gamma^{\prime}$, similar to (1), the scattered field is given by

$$
\begin{gathered}
u^{\prime}\left(\mathrm{r}^{\prime}, t^{\prime}\right)=A^{\prime} \quad \sum_{m=-\infty}^{\infty} i^{m} A_{m} H_{m}\left(k^{\prime} r^{\prime}\right) e^{i m \phi^{\prime}-i \omega^{\prime} \iota^{\prime}}, \\
x^{\prime}=\gamma(x-v t), \quad y^{\prime}=y, \quad z^{\prime}=z, \\
t^{\prime}=\gamma\left(t-\frac{v x}{c^{2}}\right), \quad \gamma=\left(1-\beta^{2}\right)^{-1 / 2}, \quad \beta=v / c,
\end{gathered}
$$

where $c$ is the velocity of light in free space, and

$$
\frac{A^{\prime}}{A}=\frac{\omega^{\prime}}{\omega}=\frac{k^{\prime}}{k}=\gamma(1-\beta \cos \alpha)
$$

describes the transformation of the amplitude, frequency and propagation constant respectively, of the incident wave, whose $\mathbf{E}$ or $\mathbf{H}$ field is polarized parallel to the axis of the cylinder. Here $\alpha$ is the direction of propagation of the incident wave in $\Gamma$. is $[8]$

The scattered field measured in $\Gamma$ but still expressed in terms of $\Gamma^{\prime}$ coordinates $\mathrm{r}^{\prime}, t^{\prime}$

$$
\begin{aligned}
u\left(\mathrm{r}^{\prime} t^{\prime}\right) & =A^{\prime} \gamma \sum_{m=-\infty}^{\infty} i^{m} C_{m} H_{m}\left(k^{\prime} r^{\prime}\right) e^{i m \phi^{\prime}-i \omega^{\prime} \mathbf{\prime}^{\prime}} \\
C_{m} & =A_{m}+\beta\left(A_{m+1}+A_{m-1}\right) / 2
\end{aligned}
$$


From the fact that (37) and (1) have the same structure, it follows that if $\left.\partial_{\phi} u\right|_{\phi=0},\left.u\right|_{\phi=0}$ are expressed in terms of $\Gamma^{\prime}$ coordinates $\mathbf{r}^{\prime}, t^{\prime}$, then (15) is valid in terms of $D_{e}\left(B_{r^{\prime}} ; \phi^{\prime}\right)$, $D_{0}\left(B_{r^{\prime}} ; \phi^{\prime}\right)$. Hence this representation is very convenient for velocity-dependent problems, since by using one probe at rest on $\phi=0$ and measuring the field in $\Gamma$ as a function of time, we get the same information gathered by an array of probes along $\phi=0$ in $\Gamma^{\prime}$.

\section{REFERENCES}

[1] V. Twersky, Scattering of waves by two objects, in Electromagnetic waves, Univ. of Wisconsin Press, Madison, Wisc., 1962, pp. 361-389

[2] J. E. Burke, D. Censor and V. Twersky, Exact inverse-separation series for multiple scaitering in two dimensions, J. Acoust. Soc. Amer. 37, 5-13 (1965)

[3] V. Twersky, Multiple scattering of electromagnetic waves by arbitrary configurations, J. Mathematical Phys. 8, 589-610 (1967)

[4] V. Twersky, Multiple scattering by arbitrary configurations in three dimensions, J. Mathematical Phys. 3, 83-91 (1962)

[5] W. E. Hobson, The theory of spherical and ellipsoidal harmonics, Chelsea, New York, 1965, p. 21

[6] E. W. Hobson, see ref. 5, p. 13

[7] J. A. Stratton, Electromagnetic theory, McGraw-Hill, New York, 1941, p. 563ff

[8] D. Censor, Scattering in velocity-dependent systems, D. Sc. Thesis, submitted to the Senate of the Technion-Israel Institute of Technology, Haifa, Israel, January 1967 (Hebrew)

[9] D. Censor, Scattering by moving objecis and scattering in moving media, Proc. Sixth National Convention of Electrical and Electronics Engineers in Israel, Tel-Aviv, 1968, pp. 466-480

[10] D. Censor, On scattering of E.M. waves in uniformly moving media, Publications of the Department of Environmental Sciences, Tel-Aviv University, no. 70-602, 1969; J. Mathematical Phys. 11, 1968$1976(1970)$ 